\title{
Thermomechanical Waves in SMA Patches under Small Mechanical Loadings
}

\author{
Linxiang Wang and Roderick V.N. Melnik \\ MCI, Faculty of Science and Engineering, \\ University of Southern Denmark, \\ Sonderborg, DK-6400, Denmark
}

\begin{abstract}
D thermo-mechanical waves in SMA (Shape Memory Alloys) patches are simulated with a model derived for a special case of material transformations. The mathematical model includes the coupling effect between thermal and mechanical fields. It is shown that the classical 1D Falk dynamical model of SMA is a special case of the formulated 2D model. The differential algebraic approach is adopted for the numerical analysis. Computational experiments are carried out with small distributed mechanical loadings to analyze thermo-mechanical waves and coupling effects. Numerical results from 2D structures are compared with its $1 \mathrm{D}$ analog which is already been verified.
\end{abstract}

Keywords: Nonlinear waves, thermo-mechanics coupling, differentialalgebraic solver.

\section{Introduction}

A better understanding of the dynamics of SMA is an important task in many areas of applications. However, even for the one dimensional case, the analysis of this dynamics is quite involved due to martensitic phase transformation and a strongly nonlinear pattern of interaction between mechanical and thermal fields (132 and references therein). It is not trivial either to analyze numerically the thermo-mechanical interaction even when the phase transformation is not included, because the strong nonlinearity and thermo-mechanical coupling are still there. For a number of practical applications, the understanding of the dynamics of SMA structures with dimensions higher than one is required, which makes the nonlinear thermo-mechanical waves in higher dimensions essential for the applications.

Many instructive investigations have been carried out to understand dynamics during the process of martensitic phase transition. They provided a firm background for the application development, in particular inthe one-dimensional cases where the model for shape memory alloysis usually based on the LandauGinzburg free energy function (1235 and references therein). Although various approximations to the free energy function have been proposed in both one dimensional and three dimensional cases ([516] and references therein), results of two or three-dimensional cases are rarely available in the literature in the context 
of modelling the thermo-mechanical waves and phase transformations in SMA. Recently,for the simulation of 2D microstructures in ferro-elastic materials, several free energy functions were proposed 677. Although the authors proposed an interesting approach, no thermo-mechanical coupling was discussed and only static simulation of microstructures with a fixed temperature were presented in [67].

In this paper, we propose a two-dimensional dynamical model for modelling the thermo-mechanical coupling behavior. The model has been originally formulated to describe square-to-rectangular phase transformations in materials with shape memory effects. However, the phase transformations will not be discussed in this contribution. Our major emphasis here will be given to the first step in validation of the model: we will investigate the thermomechanical behavior of 2D SMA structures in the case of small mechanical loadings (not large enough to induce phase transformations). In this model, a similar free energy function as that used in [10 19] is employed to construct the 2D dynamical models.

\section{The 2D Model for SMA Dynamics}

Based on conservation laws for linear momentum and energy, the system describing coupled thermo-mechanical wave interactions for the first order phase transitions in a two dimensional SMA structure can be written as follows [16]14]

$$
\begin{aligned}
& \rho \frac{\partial^{2} u_{i}}{\partial t^{2}}=\nabla_{x} \cdot \boldsymbol{\sigma}+f_{i}, \quad i, j=1,2, \\
& \rho \frac{\partial e}{\partial t}-\boldsymbol{\sigma}^{T}:(\nabla \mathbf{v})+\nabla \cdot \mathbf{q}=g
\end{aligned}
$$

where $\rho$ is the density of the material, $\mathbf{u}=\left.\left\{u_{i}\right\}\right|_{i=1,2}$ is the displacement vector, $\mathbf{v}=\partial \mathbf{u} / \partial t$ is the velocity vector, $\boldsymbol{\sigma}=\left\{\sigma_{i j}\right\}$ is the stress tensor, $\mathbf{q}$ is the heat flux, $e$ is the internal energy, $\mathbf{f}=\left(f_{1}, f_{2}\right)^{T}$ and $g$ are mechanical and thermal loadings, respectively. Let $\phi$ be the free energy function of a thermo-mechanical system described by (1). Then, the stress and the internal energy are connected with the free energy function $\phi$ by the following relationships:

$$
\boldsymbol{\sigma}=\rho \frac{\partial \phi}{\partial \boldsymbol{\eta}}, \quad e=\phi-\theta \frac{\partial \phi}{\partial \theta},
$$

where $\theta$ is the temperature, and the Cauchy-Lagrangian strain tensor $\boldsymbol{\eta}$ is given by its components as follows(with the repeated-index convention used):

$$
\eta_{i j}(\mathbf{x}, t)=\left(\frac{\partial u_{i}(\mathbf{x}, t)}{\partial x_{j}}+\frac{\partial u_{j}(\mathbf{x}, t)}{\partial x_{i}}\right) / 2,
$$

where $\boldsymbol{x}$ is the coordinates of a material point in the domain of interest.

In the two-dimensional case, the square-to-rectangular transformations could be regarded as a 2D analog of the cubic-to-tetragonal and tetragonal-toorthorhombic transformations observed in general three-dimensional cases 6] 
7]. It was established earlier for this kind of transformations that the free energy function $\phi$ can be constructed by taking advantage of a Landau free energy function $F_{L}$ [67]. Here, a simple free energy function is chosen for our purpose following the proposal in ([106]7,17,8] and references therein):

$$
\phi=-c_{v} \theta \ln \theta+\frac{1}{2} a_{1} e_{1}^{2}+\frac{1}{2} a_{3} e_{3}^{2}+F_{L}, \quad F_{L}=\frac{1}{2} a_{2}\left(\theta-\theta_{0}\right) e_{2}^{2}-\frac{1}{4} a_{4} e_{2}^{4}+\frac{1}{6} a_{6} e_{2}^{6},
$$

where $c_{v}$ is the specific heat constant, $\theta_{0}$ is the reference temperature for transformation, $a_{i}, i=1,2,3,4,6$ are the material-specific coefficients, and $e_{1}, e_{2}, e_{3}$ are dilatational, deviatoric, and shear components of strain, respectively. The later are defined as follows:

$$
e_{1}=\left(\eta_{11}+\eta_{22}\right) / \sqrt{2}, \quad e_{2}=\left(\eta_{11}-\eta_{22}\right) / \sqrt{2}, e_{3}=\left(\eta_{12}+\eta_{21}\right) / 2 .
$$

By substituting the free energy function defined by Eq. (4) into the model (1) (3)), the following coupled system of equations are obtained. For the numerical analysis, the displacement are replaced by the strains in the governing equations:

$$
\begin{aligned}
\frac{\partial^{2} u_{1}}{\partial t^{2}} & =\frac{\sqrt{2}}{2} \frac{\partial}{\partial x}\left(a_{1} e_{1}+a_{2}\left(\theta-\theta_{0}\right) e_{2}-a_{4} e_{2}^{3}+a_{6} e_{2}^{5}\right)+\frac{1}{2} \frac{\partial}{\partial y}\left(a_{3} e_{3}\right)+f_{1}, \\
\frac{\partial^{2} u_{2}}{\partial t^{2}} & =\frac{1}{2} \frac{\partial}{\partial x}\left(a_{3} e_{3}\right)+\frac{\sqrt{2}}{2} \frac{\partial}{\partial x}\left(a_{1} e_{1}-a_{2}\left(\theta-\theta_{0}\right) e_{2}+a_{4} e_{2}^{3}-a_{6} e_{2}^{5}\right)+f_{2}, \\
c_{v} \frac{\partial \theta}{\partial t} & =k\left(\frac{\partial^{2} \theta}{\partial x^{2}}+\frac{\partial^{2} \theta}{\partial y^{2}}\right)+\frac{\sqrt{2}}{2} a_{2} \theta e_{2} \frac{\partial e_{2}}{\partial t}+g .
\end{aligned}
$$

The above mathematical model is expected be able to capture the thermomechanical interactions and phase transformations in the 2D SMA structures. However, as we have mentioned before, the phase transformations are excluded from our analysis here. We aim only at initial validation of the model with relatively simple model examples. For this purpose, only a small constant mechanical loading, which is not strong enough to induce phase transformations, is applied to the above model. Then, we compare our results to the results obtained with the corresponding 1D model. This 1D mathematical model is the well known Falk model 414]. As we pointed out in [18], this model can be obtained directly by a reduction of the above $2 \mathrm{D}$ model:

$$
\begin{aligned}
\rho \frac{\partial^{2} u}{\partial t^{2}} & =\frac{\partial}{\partial x}\left(k_{1}\left(\theta-\theta_{1}\right) \frac{\partial u}{\partial x}-k_{2}\left(\frac{\partial u}{\partial x}\right)^{3}+k_{3}\left(\frac{\partial u}{\partial x}\right)^{5}\right)+F, \\
c_{v} \frac{\partial \theta}{\partial t} & =k \frac{\partial^{2} \theta}{\partial x^{2}}+k_{1} \theta \frac{\partial u}{\partial x} \frac{\partial v}{\partial t}+G,
\end{aligned}
$$

where $k_{1}, k_{2}, k_{3}, c_{v}$ and $k$ are re-normalized material-specific constants, $\theta_{1}$ is the reference temperature for 1D martensitic transformations, and $F$ and $G$ are distributed mechanical and thermal loadings.

\section{$3 \quad$ Numerical Methodology}

For the convenience of numerical simulation, the $2 \mathrm{D}$ model is re-written in the following form in terms of the dilatational and deviatoric strains introduced above: 


$$
\begin{aligned}
& \frac{\partial e_{1}}{\partial t}=\left(\frac{\partial v_{1}}{\partial x}+\frac{\partial v_{2}}{\partial y}\right) / \sqrt{2}, \quad \frac{\partial e_{2}}{\partial t}=\left(\frac{\partial v_{1}}{\partial x}-\frac{\partial v_{2}}{\partial y}\right) / \sqrt{2} \\
& \frac{\partial v_{1}}{\partial t}=\frac{\partial \sigma_{11}}{\partial x}+\frac{\partial \sigma_{12}}{\partial y}+f_{1}, \quad \frac{\partial v_{2}}{\partial t}=\frac{\partial \sigma_{12}}{\partial x}+\frac{\partial \sigma_{22}}{\partial y}+f_{2} \\
& c_{v} \frac{\partial \theta}{\partial t}=k\left(\frac{\partial^{2} \theta}{\partial x^{2}}+\frac{\partial^{2} \theta}{\partial y^{2}}\right)+\frac{\sqrt{2}}{2} a_{2} \theta e_{2} \frac{\partial e_{2}}{\partial t}+g \\
& \sigma_{11}=\frac{\sqrt{2}}{2}\left(a_{1} e_{1}+a_{2}\left(\theta-\theta_{0}\right) e_{2}-a_{4} e_{2}^{3}+a_{6} e_{2}^{5}\right) \\
& \sigma_{12}=\sigma_{21}=\frac{1}{2}\left(a_{3} e_{3}\right) \\
& \sigma_{22}=\frac{\sqrt{2}}{2}\left(a_{1} e_{1}-a_{2}\left(\theta-\theta_{0}\right) e_{2}+a_{4} e_{2}^{3}-a_{6} e_{2}^{5}\right) .
\end{aligned}
$$

The above formulated model should be simulated together with the compatibility relations in terms of the strains [7]:

$$
\frac{\partial^{2} e_{1}}{\partial x_{1}^{2}}+\frac{\partial^{2} e_{1}}{\partial x_{2}^{2}}-\sqrt{8} \frac{\partial^{2} e_{3}}{\partial x_{1} \partial x_{2}}-\frac{\partial^{2} e_{2}}{\partial x_{1}^{2}}+\frac{\partial^{2} e_{2}}{\partial x_{2}^{2}}=0 .
$$

The 2D model given by Eq. (8) is a differential-algebraic system, it is obtained by keeping the constitutive relations as algebraic equations while the stress components are kept as independent variables to be solved for. The idea of simulating the thermo-mechanical waves by the differential-algebraic approach is stimulated by the idea of 12 where the same approach was adopted for the simulation of phase transformations in SMA. We note that to deal with a strong (cubic and quintic) nonlinearities, a smoothing procedure similar to that proposed in [15] has been employed. In particular, we have used the following expansions:

$$
\epsilon^{3}=\frac{1}{4} \sum_{i=0}^{3} \epsilon_{n}^{i} \epsilon_{n-1}^{3-i} ; \quad \epsilon^{5}=\frac{1}{6} \sum_{i=0}^{5} \epsilon_{n}^{i} \epsilon_{n-1}^{6-i},
$$

where $\epsilon_{n}$ is the strain on the current time layer $n$ while $\epsilon_{n-1}$ is the strain on the previous time layer $n-1$ (For 1D case, $\epsilon=\partial u / \partial x$, while for 2D problem, $\left.\epsilon=e_{2}\right)$. We summarise this smoothing procedure as follows: Nonlinear terms are averaged here in the Steklov sense, so that for nonlinear function $f(\epsilon)$ (in particular, $\epsilon^{3}$ and $\left.\epsilon^{5}\right)$, averaged in the interval $\left[\epsilon_{n-1}, \epsilon_{n}\right]$, we have

$$
g\left(\epsilon_{n-1}, \epsilon_{n}\right)=\frac{1}{\epsilon_{n}-\epsilon_{n-1}} \int_{\epsilon_{n-1}}^{\epsilon_{n}} f(\eta) d \eta, \quad \epsilon_{n-1}=\epsilon\left(t_{n-1}\right), \quad \epsilon_{n}=\epsilon\left(t_{n}\right) .
$$

System (8) is discretized on a staggered grid. There are totally eight variables need to be solved for the two dimensional problems. The variables $e_{1}, e_{2}, \theta, \sigma_{11}$, $\sigma_{12}, \sigma_{22}$ are discretized at the same nodes, while velocity components $v_{1}$ and $v_{2}$ are computed at the flux points. For the time integration, the backward differentiation formula methodology is applied to obtain the numerical solution of the problem. 

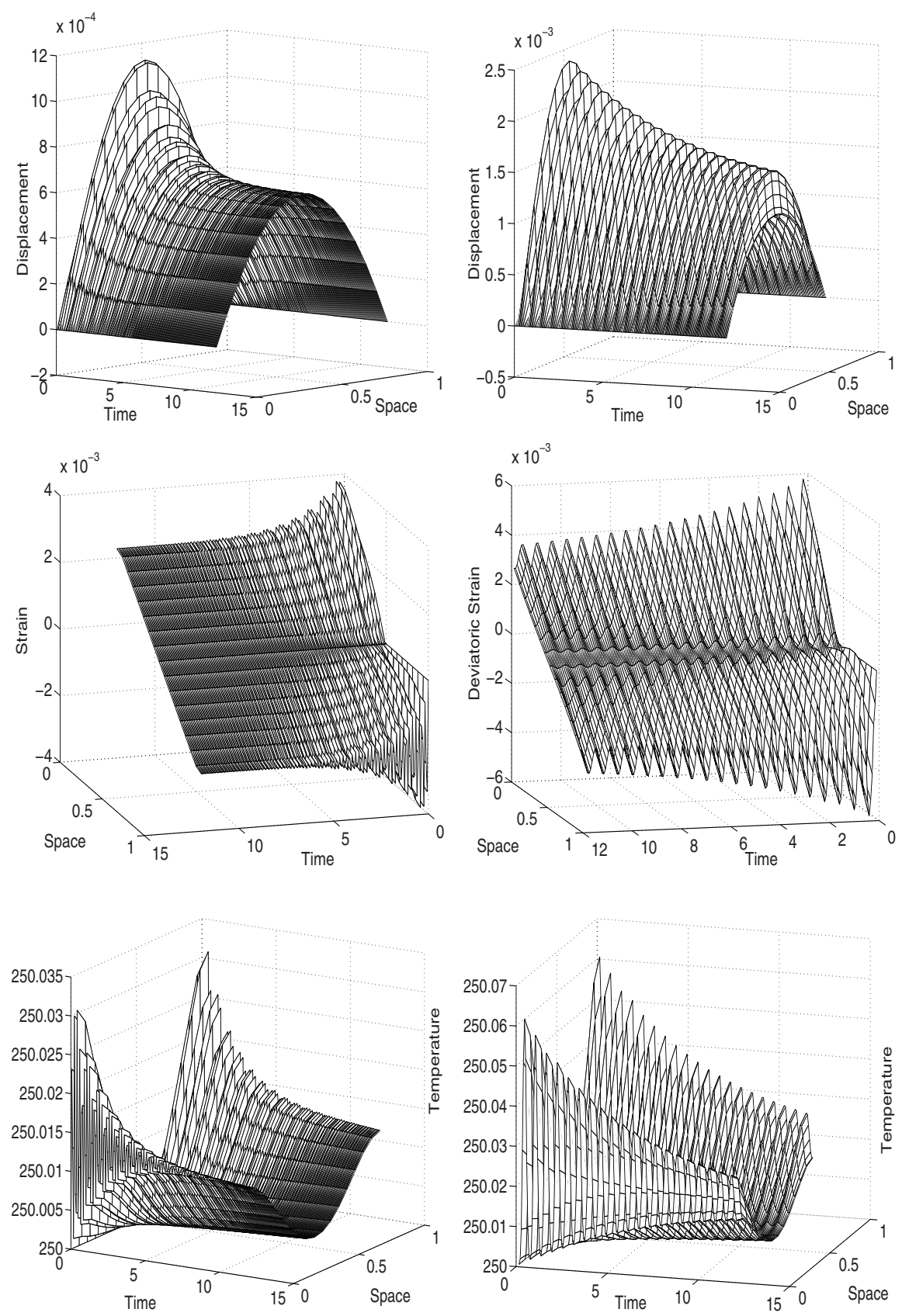

Fig. 1. Thermo-mechanical waves in SMA caused by small mechanical loadings. Left and right columns present the results obtained with the $1 \mathrm{D}$ and $2 \mathrm{D}$ models, respectively. 

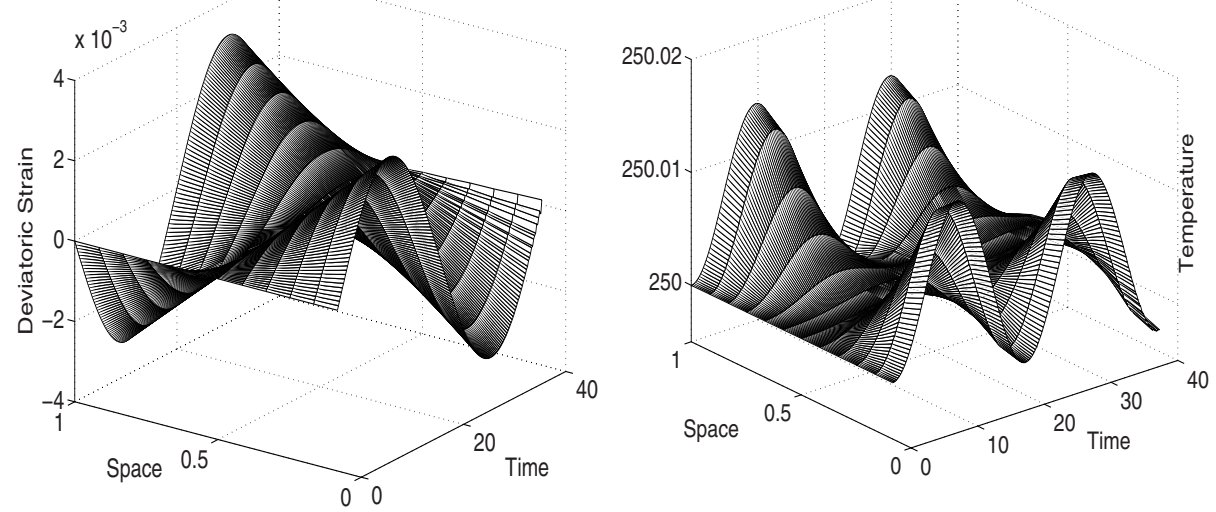

Fig. 2. Thermo-mechanical waves in SMA patch caused by varying mechanical loadings.

\section{Numerical Results}

Here, we demonstrate first the similarity of the response of $1 \mathrm{D}$ and 2D SMA structures under a small constant mechanical loadings, based on the numerical simulation. All simulations are carried out here on the same material, which is $\mathrm{Au}_{23} \mathrm{Cu}_{30} \mathrm{Zn}_{47}$. For this specific material, all necessary physical parameters are taken the same as in [12].

The first simulation is performed in a long thin $\mathrm{Au}_{23} \mathrm{Cu}_{30} \mathrm{Zn}_{47}$ strip of length $L=1 \mathrm{~cm}$ based on the $1 \mathrm{D}$ model given by Eq.(7). The initial conditions for this simulation are taken as: $\theta^{0}=250^{\circ} K, \epsilon^{0}=v^{0}=s^{0}=0$ where $\epsilon=\partial u / \partial x$, $s=k_{1}\left(\theta-\theta_{1}\right) \epsilon-k_{2} \epsilon^{3}+k_{3} \epsilon^{5}, v=\partial u / \partial t$. Boundary conditions are taken as pinned-end mechanically and insulated thermally. The distributed mechanical loading is assumed constant as $F=100 \mathrm{~g} /\left(\mathrm{ms}^{3} \mathrm{~cm}\right)$, and there are no distributed thermal loadings. There are 17 nodes used for $\epsilon, s$ and $\theta$ in the computational domain and 16 nodes are used for $v$ approximated at flux points. Time span of the simulation is $[0,12]$ and time stepsize is set to 0.00025 . Displacement, strain, and temperature distributions in the SMA strip are presented in Fig.1, from top to bottom on the left side. The MATLAB program for this case is already validated in [1812, where the phase transformations are also investigated using the same model and algorithm.

The second simulation is performed in a $\mathrm{Au}_{23} \mathrm{Cu}_{30} \mathrm{Zn}_{47}$ patch with an area of $1 \times 0.4 \mathrm{~cm}^{2}$. For this simulation, the coefficients in the 2D model are taken as follows: $a_{2}=k_{1}, a_{3}=k_{2}, a_{4}=k_{3}, a_{1}=k_{1}, a_{3}=2 k_{1}$, and all other coefficients are taken the same value as those in the $1 \mathrm{D}$ case. The initial temperature is also 
$250^{\circ}$ while all other variables are initially zero. Boundary conditions are taken as follows:

$$
\begin{array}{ll}
\frac{\partial \theta}{\partial x}=0, & \frac{\partial u_{2}}{\partial x}=0, \quad u_{1}=0, \quad \text { on left and right boundaries } \\
\frac{\partial \theta}{\partial y}=0, & \frac{\partial u_{1}}{\partial y}=0, \quad u_{2}=0, \quad \text { on top and bottom boundaries. }
\end{array}
$$

Because the displacement components are already replaced by the strains in Eq. (8), the above boundary conditions are enforced in terms of velocities when the mechanical boundary conditions are concerned.

There are 17 nodes used in the $\mathrm{x}$-direction and 7 in the $\mathrm{y}$-direction in the computational domain. As before, $v_{1}$ and $v_{2}$ are computed at the flux points. The same time span and stepsize are used as those in the 1D case. The distributed mechanical loading is enforced only in the x-direction as: $f_{1}=100 \mathrm{~g} /\left(\mathrm{ms}^{3} \mathrm{~cm}\right)$. Displacement, and strain, and temperature distributions on the line $y=0.2 \mathrm{~cm}$ (the central horizontal line) in the SMA patch are presented in Fig.1, from top to bottom on the right side. It is shown clearly from the comparison of strain and temperature evolutions that, when the oscillations in the other direction can be neglected, the 2D SMA structure respond to the mechanical loadings in the same way as its $1 \mathrm{D}$ analog. It is also seen from the computation that the oscillations in $1 \mathrm{D}$ case are dissipated much faster than those in the 2D case. This implies that there might be a numerical instability problem in $2 \mathrm{D}$ simulations when the phase transformations take place due to the slow dissipation of oscillations. This comparison can be used as an initial validation of the $2 \mathrm{D}$ model.

The third numerical experiment is to investigate the dynamical thermomechanical response of the SMA patch under varying distributed mechanical loadings, but still small enough not to induce phase transformations. The SMA patch is now covers an area of $1 \times 1 \mathrm{~cm}^{2}$, and there are 12 nodes in each direction. The loadings employed for this simulation are: $f_{1}=200 \sin (20 \pi t / 40) \mathrm{g} /\left(\mathrm{ms}^{3} \mathrm{~cm}\right)$ in the x-direction, and $f_{2}=f_{1}$ in the y-direction. The time span for this simulation is $[0,40]$ (one period of loading) and the time stepsize is set at $4 \times 10^{-5}$. All other simulation conditions and parameters are the same as those in the second case. The deviatoric strain and temperature distributions are presented in Fig.2. The simulation shows clearly that both temperature and strain field are driven periodically by the distributed mechanical loading because of the thermomechanical coupling. Under such a small loading, the SMA patch behaves just like a classical thermoelastic material.

Based on these three numerical experiments, we conclude that the formulated $2 \mathrm{D}$ model is able to capture the thermo-mechanical coupling in SMA. It gives the same prediction about the dynamical behavior of the SMA structure as that of the $1 \mathrm{D}$ model, when oscillations in one of the directions are negligible.

\section{References}

1. Ball, R., James, M.: Fine phase mixtures as minimizers of energy. Archive. Rat. Mech. Anal. 100 (1988) 13-52. 
2. Birman,V.: Review of mechanics of shape memory alloys structures, Appl.Mech.Rev. 50 (1997) 629-645.

3. Bunber, N.: Landau-Ginzburg model for a deformation-driven experiment on shape memory alloys. Continuum Mech. Thermodyn. 8 (1996) 293-308.

4. Falk, F.: Model free energy, mechanics, and thermomechanics of shape memory alloys. Acta Metallurgica, 28, (1980) 1773-1780.

5. Falk, F., Konopka, p.: Three-dimensional Landau theory describing the martensitic phase transformation of shape memory alloys. J.Phys.:Condens.Matter 2 (1990) 61-77.

6. Ichitsubo, T., Tanaka, K., Koiva, M.,Yamazaki, Y.: Kinetics of cubic to tetragonal transformation under external field by the time-dependent Ginzburg-Landau approach. Phys. Rev. B 62 (9) (2000) 5435-5441.

7. Jacobs, A.: Landau theory of structures in tetragonal-orthorhombic ferro-elastics. Phys. Rev. B 61 (10) (2000) 6587-6595.

8. Kartha, S.: Disorder-driven pretransitional tweed pattern in martensitic transformations. Phys. Rev. B 52 (2) (1995) 803-823.

9. Luskin, M.: On the computational of crystalline microstructure. Acta Numerica $\mathbf{5}$ (1996) 191-256.

10. Lookman, T., Shenoy, S., Rasmusseh, D., Saxena, A., Bishop, A.: Ferro-elastic dynamics and strain compatibility. Phys. Rev. B 67(2003) 024114.

11. Matus, P., Melnik, R., Wang, L., Rybak, I.: Application of fully conservative schemes in nonlinear thermoelasticity: Modelling shape memory materials. Mathematics and Computers in Simulation (to appear)

12. Melnik, R., Roberts, A., Thomas, K.: Phase transitions in shape memory alloys with hyperbolic heat conduction and differential algebraic models. Computational Mechanics 29 (1) (2002) 16-26.

13. Melnik, R., Roberts, A., Thomas, K.: Coupled thermomechanical dynamics of phase transitions in shape memory alloys and related hysteresis phenomena. Mechanics Research Communications 286 (2001) 637-651.

14. Melnik, R., Robert, A., Thomas, K.: Computing dynamics of Copper-based SMA via center manifold reduction models. Computational Material Science 18(2000) 255-268.

15. Niezgodka, M., Sprekels, J.: Convergent numerical approximations of the thermomechanical phase transitions in shape memory alloys. Numerische Mathematik 58(1991) 759-778.

16. Pawlow, I.: Three dimensional model of thermomechanical evolution of shape memory materials. Control and Cybernetics 29 (2000) 341-365.

17. Saxena, A., Bishop, A., Shenoy, S., Lookman, T.: Computer simulation of martensitic textures. Computational Materials Science 10 (1998) 16-21.

18. Wang, L., Melnik, R.: Nonlinear coupled thermomechanical waves modelling shear type phase transformation in shape memory alloys. in Mathematical and Numerical Aspects of Wave Propagation, Eds.G.C.Cohen, et al,Springer,723-728 (2003). 